http://jmscr.igmpublication.org/home/ ISSN (e)-2347-176x ISSN (p) 2455-0450

crossref DOI: https://dx.doi.org/10.18535/jmscr/v7i7.96

\title{
A Prospective Observational Study on Prevalence, Management and Estimation of Quality of Life in Patients with Low Back Pain
}

\author{
Authors \\ Syeda Zohra Altaf', Madapathi Soujanya ${ }^{1}$, Kotte Samanth ${ }^{1}$, Kadarla Rohith Kumar ${ }^{* 2}$ \\ ${ }^{1}$ Department of Pharmacy Practice, Sree Chaitanya Institute of Pharmaceutical Sciences, Thimmapur, \\ Karimnagar, Telangana, India \\ ${ }^{* 2}$ Asst Professor, Department of Pharmacy Practice, Sree chaitanya Institute of Pharmaceutical Sciences, \\ Thimmapur, Karimnagar, Telangana, India
}

*Corresponding Author

Dr Kadarla Rohith Kumar

Asst Professor, Department of Pharmacy Practice, Sree chaitanya Institute of Pharmaceutical Sciences, Thimmapur, Karimnagar, Telangana, India

\begin{abstract}
Background: $L B P$ is defined as a pain, muscle tension or discomfort located below the margin of the 12th rib and above the inferior gluteal fold, with or without leg pain ${ }^{[1] .}$ It is defined as chronic when it persists for 12 weeks or more. It is the pain that appears in the lumbosacral area, and between the inferior rib cage and the sacrum region and is caused by a muscular-skeletal syndrome; it refers to alterations related to the lumbar vertebrae and the structures of the soft tissues \{muscles, ligaments, nerves and intervertebral discs\}. Low back pain is one of the most common health problems among all the population of the world. Men and women are equally reported to be affected by this condition. This study was hence conducted to assess the prevalence, management and estimation of quality of life in patients with LBP.
\end{abstract}

Methods: Patient prescriptions and medical records were studied to obtain demographic details. Other information is obtained from patient which include lifestyle, working status, duration of condition, presence of other comorbid conditions, social habits (alcohol consumption, smoking). Data from X-ray, MRI scan was obtained.

Results: The most common age group to be affected among the males and females was 41-50 years of age, where males affected are 26\% and females affected are 35.04\%. Most ofthe patients had LBP due to hard work and then followed by sitting for long period of time and followed by patients who are overweight. Patients with LBP are usually treated with analgesics, vitamin D supplement, calcium supplement and some patients are also treated with methylcobalamin.

Conclusion: our study concludes that LBP is prevalent in the town of Karimnagar among middle aged and old aged patients and the incidence is more among males than females

Keywords: Low back pain, prevalence, management, quality of life.

\section{Introduction}

Low back pain is one of the most common health problems among all the population of the world. Most people experience low back pain at some point of theirlives ${ }^{2}$. Although most of the people recover from the pain quickly, the disability which is the result of such pain most often leads to a limited range of activity among the adults, which 
is only next to arthritis. ${ }^{3}$ The prevalence of low back pain has been reported among many people especially when resulting from work related and occupational activities. ${ }^{4,5}$

Low back pain is defined as a pain, muscle tension or discomfort located below the margin of the 12th rib and above the inferior gluteal fold, with or without leg pain ${ }^{[1]}$. It is defined as chronic when it persists for 12 weeks or more. It is the pain that appears in the lumbosacral area, and between the inferior rib cage and the sacrum region and is caused by a muscular-skeletal syndrome; it refers to alterations related to the lumbar vertebrae and the structures of the soft tissues \{muscles, ligaments, nerves and intervertebral discs \}. It usually appears along with spasms in the muscles on either side of the spinal column and in certain cases may be radiated to the gluteal area and even to the lower limbs. Chronic pain may be associated with tingling sensation or pain in the lower limbs, normally in one of them, even though it could appear in both of them, which it leads to call it sciatic. LBP symptoms can derive from many potential anatomic sources, such as nerve roots, muscle, fascial structures, bones, joints, intervertebral discs (IVDs), and organs within the abdominal cavity. Moreover, symptoms can also spawn from aberrant neurological pain processing causing neuropathic $\mathrm{LBP}^{6,7}$. LBP can also be influenced by psychological factors, such as stress, depression, and/or anxiety ${ }^{8,9}$. The lumbar pain may be intrinsic to the lumbar column the one originating in the structures forming lumbar and lumbosacral columns\} and extrinsic the one originated out of the above mentioned structures, that is in case of gynecological, kidney, sacroiliac diseases or psychosomatic symptomsz. In most of the cases this pain has a mechanic functional origin that is, due to an abnormal joint functioning of the lumbar vertebrae, also associated to other affecting factors like muscular spasms, spinal disc herniation, scoliosis, osteoarthritis, etc.

Treatment for low back pain generally depends on whether the pain is acute or chronic. In general, surgery is recommended only if there is evidence of worsening nerve damage and when diagnostic tests indicate structural changes for which corrective surgical procedures have been developed. Non pharmacological treatment includes short Rest Period, activity Modification, heat/Ice Therapy, physical therapy. Over-TheCounter Pain Medications: The most common overthe-counter (OTC) medications are aspirin, ibuprofen, naproxen, and acetaminophen. Aspirin, ibuprofen, and naproxen are anti-inflammatory medicines, which alleviate low back pain caused by a swollen nerves or muscles. Acetaminophen works by interfering with pain signals sent to the brain. Pharmacological treatment include enonsteroidal anti-inflammatory drugs (NSAIDS): ibuprofen, diclofenac, celecoxib, tramadol; Membrane stabilizers: carbamazepine, gabapentin, topiramate; Antidepressants: nortriptyline, amitriptyline. Surgical procedures include spinal fusion, laminectomy, for aminotomy, microdiscectomy.

\section{Objective}

1) To study the prevalence of cases with low back pain.

2) To determine the efficacy and tolerability of analgesics and adjuvant pain drugs administered for the management of patients with chronic low back pain.

3) To evaluate the treatment outcomes and to assess the disease condition before and after the treatment.

4) To assess the severity of pain and estimation of outcomes using pain scales of low back pain.

5) To assess/study the quality of life in patients with low back pain.

\section{Materials and Methods}

This study is conducted in a total of 252 patients for a period of 6 months in a multi-specialty hospital. It is a prospective observational study conducted to find prevalence, management and estimation of quality of life in patients with le back pain. 
Inclusion Criteria: All patients of all age groups suffering from low back pain are diagnosed and admitted in the hospital and with moderate to severe low back pain were taken into the study

Exclusion Criteria: Patients suffering with low back pain due to any history of trauma.

\section{Study procedure}

Patient prescriptions and medical records were studied to obtain demographic details. Other information was asked verbally which included lifestyle, working status, duration of condition, presence of other comorbid conditions, social habits (alcohol consumption, smoking).Data from X-ray, MRI scan was obtained.

\section{Results}

A total number of 252 patients who visited hospital with low back ache are participated in the study.

Table 1: Distribution of patients according to age and gender

\begin{tabular}{|l|c|c|c|c|c|c|}
\hline Age & Males & Percentage & Females & Percentage & Number of patients & Percentage \\
\hline $21-30$ & 13 & 9.6 & 18 & 15.4 & 31 & 12.3 \\
\hline $31-40$ & 29 & 21.5 & 20 & 17.1 & 49 & 19.4 \\
\hline $41-50$ & 35 & 26 & 41 & 35.04 & 76 & 30.15 \\
\hline $51-60$ & 42 & 31 & 25 & 21.4 & 67 & 26.6 \\
\hline $61-70$ & 16 & 11.8 & 13 & 11 & 29 & 11.5 \\
\hline
\end{tabular}

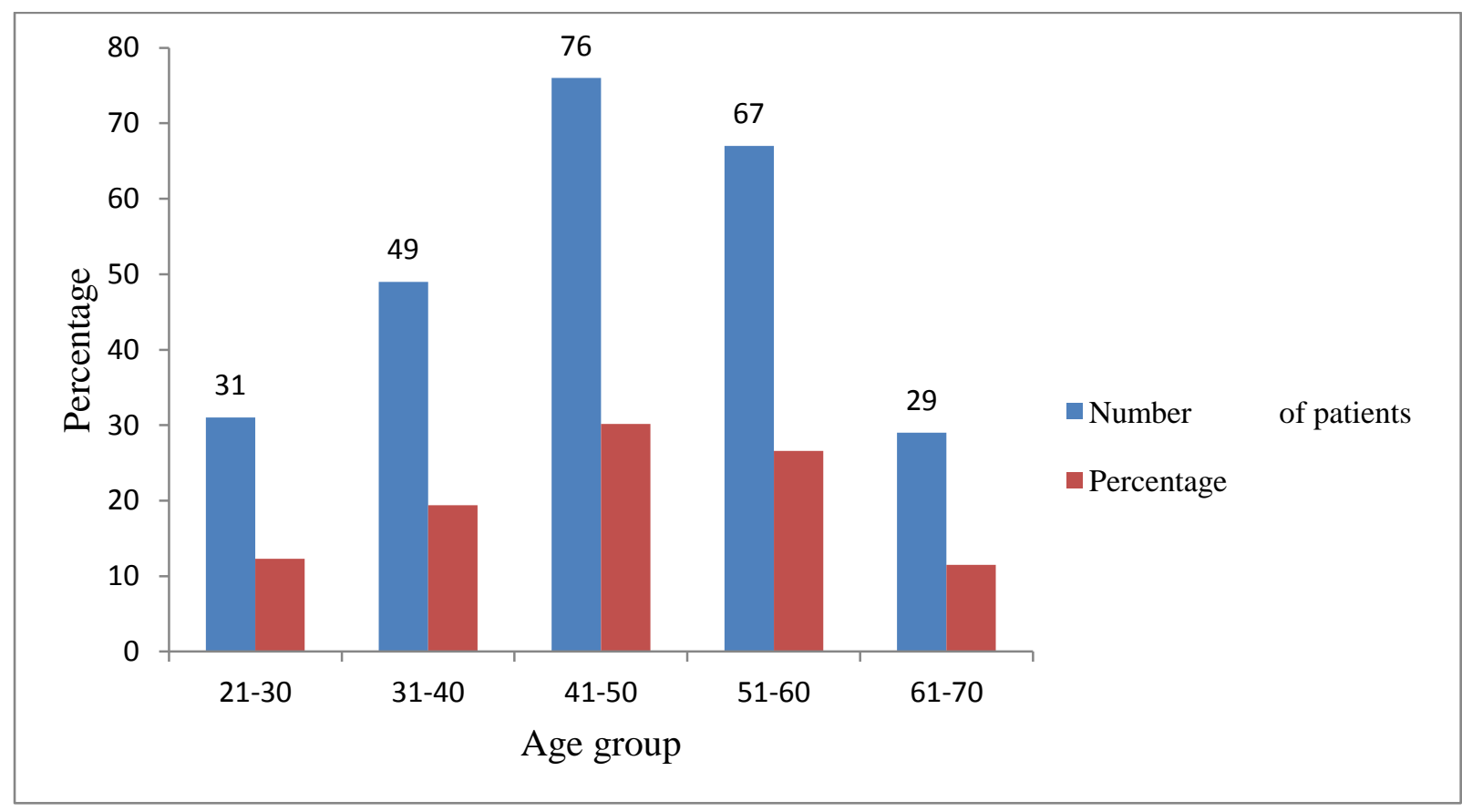

Figure 1: Distribution of patients according to age and gender

Table 2: Distribution of patients according to time and duration of pain

\begin{tabular}{|l|c|c|}
\hline Duration of pain & Number of patients & Percentage \\
\hline 3 months & 143 & 56.7 \\
\hline 6 months & 75 & 29.8 \\
\hline More than 6 months & 34 & 13.5 \\
\hline
\end{tabular}

Table 3: Distribution of patients according to risk factors

\begin{tabular}{|l|c|c|}
\hline Risk factor & Number of patients & Percentage \\
\hline Hard work & 158 & 62.6 \\
\hline Over weight & 35 & 13.9 \\
\hline Driving for long period of time & 47 & 17.1 \\
\hline Lack of strength and resistance in the muscles of spinal column & 12 & 4.8 \\
\hline
\end{tabular}




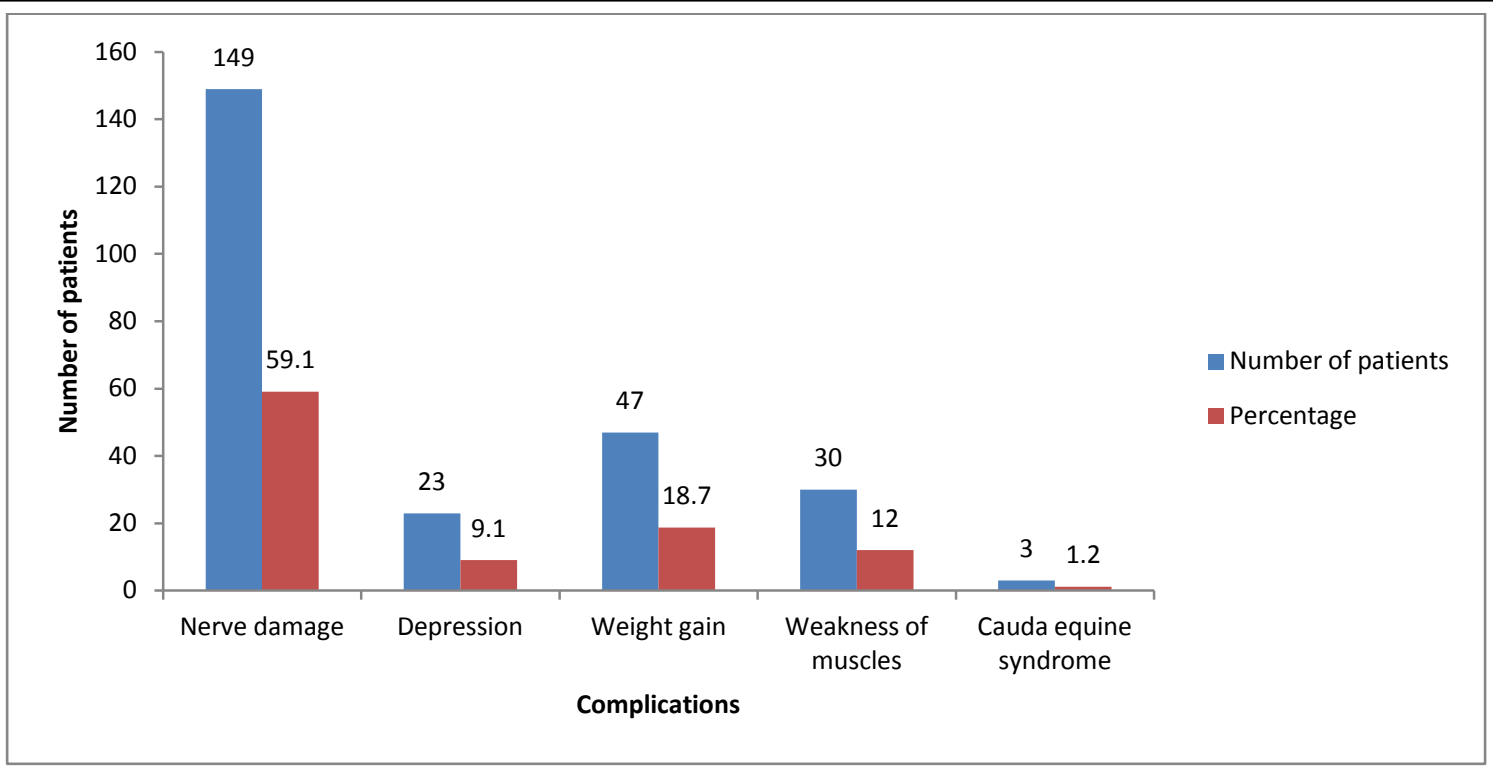

Figure 2: Distribution of patients according to complications

Table 4: Distribution of patients according to classes of drugs prescribed

\begin{tabular}{|l|c|c|}
\hline Classes & Number Of Patients & Percentage \\
\hline NSAIDS & 252 & 100 \\
\hline Vitamin D & 252 & 100 \\
\hline Calcium supplement & 252 & 100 \\
\hline Methyl cobalamine & 47 & 18.6 \\
\hline Opioids & 56 & 22.2 \\
\hline
\end{tabular}

Table 5: Distribution of patients according to drugs prescribed

\begin{tabular}{|l|c|c|}
\hline Name of the drug & Number of patients & Percentage \\
\hline Diclofenac & 132 & 52.4 \\
\hline Tramadol & 56 & 22.2 \\
\hline Pregabalin & 34 & 13.5 \\
\hline Gabapentin & 34 & 13.5 \\
\hline Amitriptylline & 12 & 4.8 \\
\hline Vitamin D & 252 & 100 \\
\hline Calcium supplements & 252 & 100 \\
\hline Ketorolac & 35 & 13.8 \\
\hline Ibuprofen & 17 & 6.7 \\
\hline Naproxen & 72 & 28.5 \\
\hline Aceclofenac & 125 & 49.6 \\
\hline
\end{tabular}

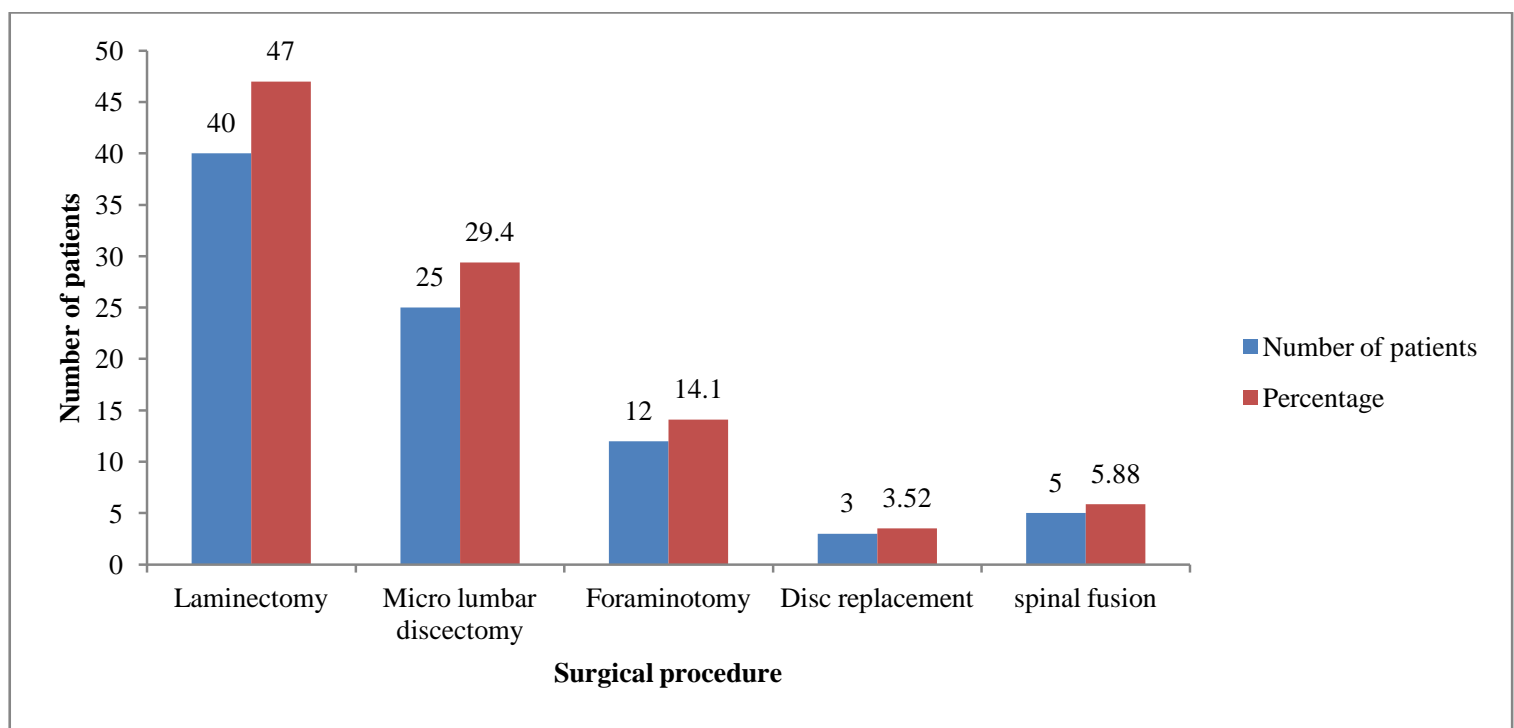

Figure 3: Distribution of patients based on surgery 
Table 6: Assessment of pain outcomes before and after management

\begin{tabular}{|l|c|c|c|c|c|}
\hline $\begin{array}{l}\text { Pain readings- } \\
\text { before treatment }\end{array}$ & $\begin{array}{c}\text { Number of } \\
\text { patients }\end{array}$ & Percentage & $\begin{array}{c}\text { Pain readings- } \\
\text { after treatment }\end{array}$ & $\begin{array}{c}\text { Number } \\
\text { of patients }\end{array}$ & Percentage \\
\hline 0 & 09 & 3.57 & 0 & 28 & 11.1 \\
\hline 1 & 13 & 5.15 & 1 & 45 & 17.85 \\
\hline 2 & 16 & 6.34 & 2 & 44 & 17.46 \\
\hline 3 & 17 & 6.74 & 3 & 36 & 14.28 \\
\hline 4 & 28 & 11.1 & 4 & 26 & 10.31 \\
\hline 5 & 16 & 6.34 & 5 & 17 & 6.74 \\
\hline 6 & 25 & 9.92 & 6 & 14 & 5.5 \\
\hline 7 & 32 & 12.69 & 7 & 19 & 7.53 \\
\hline 8 & 47 & 18.65 & 8 & 15 & 5.95 \\
\hline 9 & 39 & 15.47 & 9 & 07 & 2.7 \\
\hline 10 & 10 & 3.96 & 10 & 03 & 1.19 \\
\hline
\end{tabular}



Figure 4: Assessment of pain outcomes before management

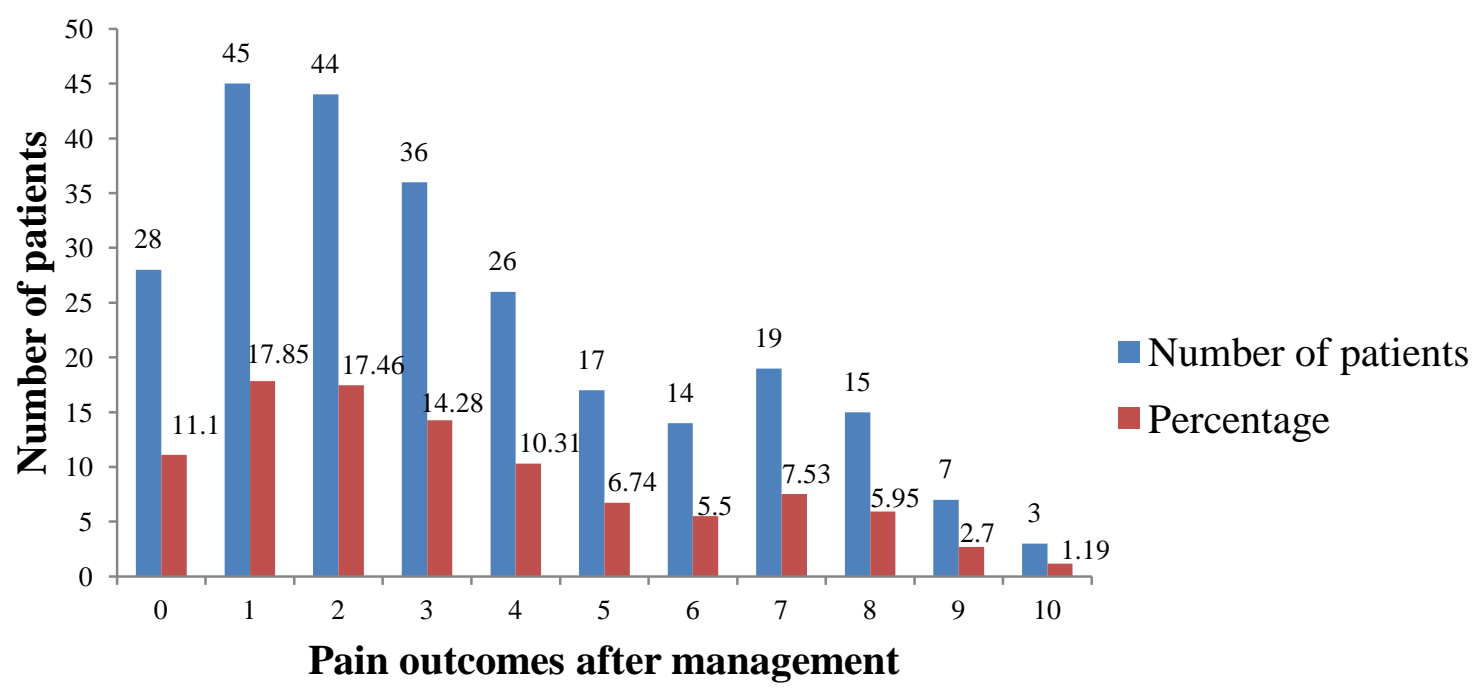

Figure 5: Assessment of pain outcomes after management 


\section{Discussion}

Low back pain is a common condition affecting many individuals at some point in their lives. The estimation is that between $5.0 \%$ and $10.0 \%$ of cases will develop chronic low back pain (CLBP), which is responsible for high treatment costs, sick leave, and individual suffering ${ }^{[10]}$. The 12 month prevalence of LBP in this study was $73.53 \%$, this is considered high. They reported high annual prevalence varying from $73 \%$ to $76 \%$ among nurses $^{[11,12]}$.

There was a significant association between sex and severity of LBP. Males reported $16.67 \%$, $5.33 \%$ and $10 \%$ for mild, moderate and severe LBP, while females reported 26.67\%, 33.33 and $8 \%$ or mild, moderate and severe LBP respectively. Generally $64.86 \%$ of the total male reported LBP while $78.46 \%$ of the total female reported $\operatorname{LBP}^{[13,14,15]}$. In our study out of the 252 patients, 135 patients were male and remaining 117 were female patients. Male patients[53.6\%] suffering from low back ache are slightly higher than that of female patients[46.4\%]. Pain ratings in work population were considerably higher than expected, with 116 providing one- month LBP ratings classified as low, 170 medium and 78 with high pain ratings. Pain ratings on the day of enrollment ranged widely with 58 rating low, and 75 providing LBP ratings of medium ${ }^{[16,17]}$.In this study population we have observed that the number of patients with the duration flow back pain for 3months is maximum i.e., 143 patients [56.7\%], followed by 75 patients [29.6\%] with the duration of pain for 6months and the number of patients with the duration of pain for more than 6 months are 34[13.5\%]. A total of 114 reported pain ratings on the day of enrollment classified as medium or high. Many randomized trials investigating opioid treatment for LBP have had minimum pain rating inclusion criteria. Trials for treatment of acute or sub acute LBP have utilized inclusion criteria of at least $4 / 10$ or $5 / 10$ pain ratings. Trials assessing chronic pain had similar requirements. Similar minimum pain requirements are used in trials investigating muscle relaxants for acute and sub acute LBP treatment. In this study, 114 participants(18.8\%) had point prevalence pain meeting the criterion of $\geq 4 / 10$ for opioid treatment on the day of enrollment and 248 $(30.0 \%)$ met that criterion over the month prior to enrollment ${ }^{[18,19,20]}$.In the study population, we estimated these varity of pain using wong baker FACES pain rating scale, it was observed that out of 252 patients the maximum number of patients with assessment of pain according to pain scale are with8 (hurtswholelot) i.e.,36.90\% and the minimum number of 9patients of 0 (nohurt) are with a frequency of $3.57 \%$.Overall prescribed drugs are, Pregabalin (82\%), Amitriptyline (64\%), Duloxetine (59\%), Tramadol (42\%), Nortiptyline (36\%), topical analgesics (75\%), Calcium \& vitamin supplements (35\%), Physical exercises and posture advices(75\%) ${ }^{[21]}$. In the study population, it was observed that the maximum number of patients are prescribed with Diclofenac, Vitamin D, Calcium supplements with a frequency of $100 \%$, followed by 56patients of $22.2 \%$ with Tramadol. Surgical procedures are quite commonly used as a treatment for chronic low back pain assumed to originate from the intervertebral disc. The study of Fritzelland colleagues $^{[22]}$ showed that fusion was more effective than conservative care. In the study of population it was observed that about 85 patients had underwent surgery. About 40patients [47.0\%] had underwent laminectomy. About 25patients [29.4\%] underwent microlumbar discectomy. About 12 patients [14.1\%] had underwent for aminotomy. About 03patients [3.52\%] had underwent disc replacement. About 05patients [5.88] had underwent spinal fixation. The study showed that mean $( \pm \mathrm{SD})$ age was $37.41( \pm 10.63)$ years and majority $(52.7 \%)$ of the LBP patients was female. The study revealed that LBP was common among married housewives and mean duration of LB P was $3.59( \pm 3.52)$ years. Majority $(55.4 \%)$ of the patients had poor QOL before intervention but after self back care intervention $\operatorname{most}(83.9 \%)$ of them had average QOL. Mean $( \pm$ SD) score of QOL after intervention 
$(57.83 \pm 8.74)$ was significantly $(t, p<0.001)$ higher to be for intervention $(49.30 \pm 11.31)$. It was also found that mean QOL score with long duration of LBP (50.526 \pm 6.844$)$ was significantly (ANOVA, $\mathrm{p}<0.001)$ lower than QOL with short duration of LBP (65.137 \pm 9.538$)$. The study also found that when severity of pain increased, mean QOL score significantly (ANOVA, $\mathrm{p}<0.001$ ) decreased after intervention. Linear regression of mean QOL and duration of LBP before intervention were $\mathrm{R}^{2}=0.146$ and after intervention were $\mathrm{R}^{2}=0.214$ ).

\section{Conclusion}

Our study concludes that, low back pain is prevalent in the town of Karimnagar among middle aged and old aged patients and the incidence is more among males than females. About $66 \%$ of patients who had underwent conservative management shows a significant improvement in quality of life. In about $32 \%$ patients who had underwent conservative management and suffering reoccurred on physical strain and needed surgery. $2 \%$ patients are unable to gain back indemnity in quality of life even after surgery for about a period of 6 weeks. Our study concludes that patients with chronic low back pain have shown a significant improvement in the quality of life after treatment. It is the responsibility of clinical pharmacist to improve quality of life by creating awareness about the modifiable risk factors in population may lead to life style modifications thereby improving their quality of life.

\section{Acknowledgement}

It is a privileged honor for us to express heartfelt thanks and immense gratitude to all the people who supported us in the completion of this project work. First and foremost we would like to express gratitude and sincere thanks to Dr. Bangari Swamy, MS, and MCH (ORTHO) FSS (NIMS) for permitting us to do clinical research at Renee Hospital. We are fortunate to have a clinical guide like him. We wish to express our sincere thanks to Dr. Habeeb uddin farman for his great support and helpful suggestions. We would wish to thank each and every one of the hospital staff member. They have given us a great support and helping hand during this study period.

\section{References}

1. Meucci RD, Fassa AG, Paniz VM, Silva MC, Wegman DH (2013) Increase of chronic low back pain prevalence in a medium-sized city of southern Brazil. BMC Musculoskelet Disord 14: 155.

2. Hoy D, Brooks P, Blyth F, Buchbinder R. The Epidemiology of low back pain. Best Pract Res Clin Rheumatol. 2010; 24(6):769-81.

3. Frank JW, Kerr MS, Brooker AS, DeMaio SE, Maetzel A, Shannon HS, et al. Disability resulting from occupational low back pain: Part I: What do we know about primary prevention? A review of the scientific evidence on prevention before disability begins. Spine. 1996; 21(24):2908-17.

4. Allan DB, Waddell G. An historical perspective on low back pain and disability. Acta. Orthopaed Scand. 1989; 60 (sup234):1-23.

5. Lis AM, Black KM, Korn H, Nordin M. Association between sitting and occupational LBP. Eur Spine J. 2007; 16(2):283-98.

6. Smart KM, Blake C,Staines A,et al.: Mechanisms based classifications of musculoskeletal pain : part 1 of 3 : symptoms and signs of central sensitization in patients with low back (+/leg)pain. Man Ther.2012;17(4):336-44.

7. Garland EL: Pain processing in the human nervous system: a selective review of nociceptive and bio behavioral pathways.PrimCare.2012; 39(3):561-71.

8. Roberts S, Evans H, Trivedi J, etal. Histology and pathology of the human intervertebral disc. J Bone Joint SurgAm.2006; 88(Suppl2):10-4. 
9. Grunhagen T, Wilde G, Soukane DM, et al. Nutrient supply and intervertebral disc metabolism.JBoneJointSurgAm.2006; 88(Supp12):30-5.

10. Standring S: Gray's Anatomy, The Anatomical Basis of Clinical Practice. $40^{\text {th }}$ edn. London: Churchill Livingstone Elsevier; 2008; 749-761.

11. Pedersen HE, Blunck CF, And Gardner E: The anatomy of lumbosacral posterior rami and meningeal branches of spinal nerve (sinu-vertebra lnerves); with an experimental study of their functions. $\mathbf{J}$ Bone Joint SurgAm.1956; 38-A (2):37791.

12. Bogduk $\mathrm{N}$ : The anatomy of the lumbar intervertebral disc syndrome. Med J Aust. 1976; 1(23):878-81.

13. Amir R, Devor M: Electrical excitability of the soma of sensory neurons is required for spike invasion of the soma, but not for through-conduction.BiophysJ.2003; 84(4):2181-91.

14. Esteban-Vasallo MD, et al. Prevalencia deenfermedad escrónicas diagnostic adasenpoblacióninmigranteyautóctona.

Gac Sanit.2009; 23(6):548-52

15. Rodrigo Dalke Meucci et al. Prevalence of chronic low back pain: systematic review2015; 49:73.

16. Knuth AG, Bacchieri G et al. Changes in physical activity among Brazilian adults over a five-year period. J Epidemiol Community Health.2009; 64(7):591-5.

17. BejiaI, Younes $M$ et al. Prevalence and factors associated to low back pain among hospital staff. Joint Bone Spine 2005; 72(3):254-9.

18. Barrero LH et al. Prevalance and physical determinants of low back pain in arural Chinese population. Spine 2006:31:272834.
19. Gilette JV, Haycock CE. Proceedings of the $18^{\text {th }}$ Conference of the Medical Aspects of Sports. Chicago: American Medical Association;

20. Rasch PJ, Burke RK. Kinesiology and applied anatomy. Philadelphia: Lea \& Febiger; 1978.

21. Louw QA et al. The prevalence of low back pain in Africa: a systematic review. BMC Musculoskeletal Disorder. 2007; 8:105.

22. Pareek A et al. Aceclofenac-tizanidine in the treatment of acute low back pain: a double-blind, double-dummy, randomized, multicentric, comparative study against aceclofenac alone. Eur Spine J.2009, 18:1836-1842. 